\title{
Probing the photoreactivity of aryl chlorides with oxygen $\uparrow$
}

\author{
José P. Da Silva, ${ }^{* a}$ Steffen Jockusch $^{b}$ and Nicholas J. Turro ${ }^{b}$ \\ Received 29th August 2008, Accepted 3rd October 2008 \\ First published as an Advance Article on the web 6th November 2008 \\ DOI: $10.1039 / \mathrm{b815039g}$
}

Molecular oxygen was used to probe the mechanism of the phototransformation of chlorobenzene and 4-chloroanisole in organic solvents. Laser flash photolysis, electron paramagnetic resonance and product distribution studies clarified the reaction mechanisms of these compounds under a wide range of conditions. The main primary photochemical reaction step is the homolytic cleavage of the $\mathrm{C}-\mathrm{Cl}$ bond to produce a triplet radical pair in the solvent cage. In non-polar solvents hydrogen abstraction, after radical diffusion, leads to reduction. In polar solvents, in addition to H-abstraction, electron transfer within the caged radical pair occurs and leads to an ion pair (phenyl cation and $\mathrm{Cl}^{-}$). In the presence of oxygen, phenyl radicals can form phenylperoxyl radicals which have a bathochromically shifted absorption, thus making the homolytic cleavage visible by flash photolysis. The peroxyl radicals can couple, leading to more polar compounds, or undergo back reaction to the phenyl radical. For concentrations of the aryl chlorides of higher than $10^{-3} \mathrm{M}$, dimerization becomes an important transformation process and occurs after reaction of the transients with ground state molecules. In addition, excimer formation is postulated to be involved in the dimerization process.

\section{Introduction}

The photoreactivity of aryl halides is a subject of long standing interest. ${ }^{1}$ In recent years, the phototransformation of these compounds has attracted attention in the effort to understand their photodissociation dynamics and also in their use for synthesis. ${ }^{2}$

The chlorine induced internal heavy atom effect makes the intersystem crossing a relatively efficient process for aryl chlorides. ${ }^{3,4}$ Theoretical studies of aryl halides indicate that more than one triplet state can be involved in the phototransformation process. ${ }^{5}$ Homolytic cleavage of the $\mathrm{C}-\mathrm{Cl}$ bond is expected to be one of the primary photochemical processes from the triplet states. This is the case in non-polar solvents. ${ }^{3}$ In polar solvents, however, the photochemistry changes and both radical and ionic processes are invoked to explain the observed final products ${ }^{3,4}$ (Scheme 1).

Some fundamental mechanistic aspects are still under discussion. ${ }^{3,4}$ The role of excimer/exciplex involvement ${ }^{3 a, 4 b}$ on the product distribution has not been extensively explored. In addition, the role of molecular oxygen on the reduction versus substitution pathways, ${ }^{3 b}$ is not completely understood. One of the major difficulties is the direct analytical detection of phenyl radicals. These intermediates are $\sigma$-radicals with almost the same $\pi$-system as the parent molecules. Laser flash photolysis studies ${ }^{6}$ showed broad transient absorption bands in the same spectral region of their precursors, which makes the formation of phenyl radicals difficult to follow by flash photolysis.

In previous studies ${ }^{3 c}$ we detected the formation of the methoxyphenylperoxyl radical after photolysis of 4-chloroanisole

${ }^{a}$ Universidade do Algarve, Faculdade de Ciências e Tecnologia, Campus de Gambelas, 8005-139, Faro, Portugal.E-mail:jpsilva@ualg.pt; Fax: 351289 800066; Tel: 351289800905

${ }^{b}$ Department of Chemistry, Columbia University, New York, NY 10027

$\dagger$ Electronic supplementary information (ESI) available: Experimental details, transient absorption spectra of $\mathbf{1}$ and $\mathbf{2}$ in ethanol (Fig. S1 and S2). See DOI: $10.1039 / \mathrm{b} 815039 \mathrm{~g}$

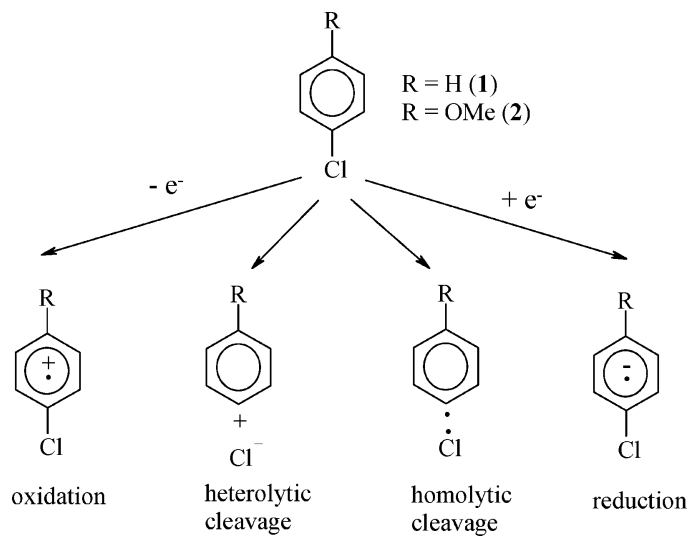

Scheme 1 Primary photochemical reactions of aryl chlorides.

(2) in cyclohexane under air saturated conditions (eqn (1)). In the present work we extend the study to chlorobenzene (1) and investigated solvent effects. In addition, we show how molecular oxygen can be used to probe the phototransformation of aryl chlorides.

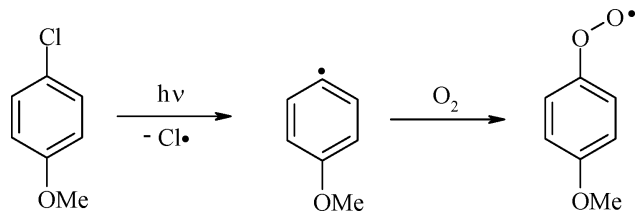

\section{Experimental}

\section{Materials}

Chlorobenzene, 4-chloroanisole, 4-chlorobiphenyl, 3-chlorobiphenyl, 2-chlorobiphenyl (Riedel-de Haën), anisole, phenol, 4-hydroxyanisole, 4,4'-dimethoxybiphenyl, 4-methoxyanisole (Aldrich), biphenyl (Fluka), methanol, acetonitrile (Merck 
Lichrosolv), cyclohexane, ethanol, benzene (Merck, p.a.), and glycerol (Sigma) were used without further treatment. Water was deionized and distilled.

\section{Methods}

Quantum yields. The phototransformation quantum yields were determined by the method described for organic pollutants and pesticides ${ }^{7,8}$ (see ESI $\dagger$ ). The quantum yields were obtained from the ratio between the phototransformation rate constants of the studied compounds and that of a reference with known phototransformation quantum yield. The reference system was chlorobenzene in methanol with a quantum yield of $0.25{ }^{4 c}$

Photoproducts. The photoproduct distributions were studied in cyclohexane, ethanol, methanol, acetonitrile under air and nitrogen atmospheres and also in glycerol/methanol mixtures (4:1) under air conditions. The studied concentrations ranged from $5 \times 10^{-5}$ to $5 \times 10^{-2} \mathrm{M}$. The quantifications in polar solvents were made by HPLC and in cyclohexane by GC-MS. In some cases the photoproducts were also studied by LC-MS. In all identification and quantification studies the samples were immediately submitted to analysis by taking an aliquot of the irradiated solution, typically $1 \mu \mathrm{l}$ for GC and $20 \mu \mathrm{l}$ for HPLC.

Electron paramagnetic resonance spectroscopy (EPR). The EPR measurements were performed at room temperature using a Brucker EMX spectrometer operating at X-band $(9.5 \mathrm{GHz})$. Solutions of 1 and $2\left(\sim 10^{-2} \mathrm{M}\right)$ were deoxygenated by argon bubbling and flown through the EPR cavity through a quartz flow cell. The sample solution was photolyzed directly inside the EPR cavity using a $300 \mathrm{~W}$ Xe-lamp (LX300UV; ILC Technology, Inc.).

Laser flash photolysis (LFP). Laser flash photolysis experiments employed the pulses from a Spectra Physics GCR-150-30 $\mathrm{Nd}$ :YAG laser (266 nm, ca. $5 \mathrm{~mJ} /$ pulse, $5 \mathrm{~ns})$ and a computer controlled system that has been described elsewhere. ${ }^{9}$ Solutions of $\mathbf{1}$ and $\mathbf{2}$ were prepared at concentrations such that the absorbance was $0.3(1 \mathrm{~cm}$ pathlength) at the excitation wavelength $(266 \mathrm{~nm})$.

Further details of the used equipment and analytical methods can be found in the ESI. $\dagger$

\section{Results and discussion}

\section{Quantum yields}

The phototransformation quantum yields of chloroaromatic compounds depend on the solvent and on the concentration of dissolved oxygen (Table 1).

The phototransformation quantum yield of $\mathbf{1}$ in cyclohexane in the absence of oxygen is in agreement with the reported quantum yield by Bunce et al..$^{3 a}$ at infinite dilution $(0.50-0.54)$. This agreement validates our experimental procedure and confirms the absence of concentration effects, which are known to reduce phototransformation quantum yields. It is reported that at higher concentrations of 1 the quantum yield is reduced due to excimer formation. ${ }^{3 a}$ The observed quantum yields for $\mathbf{2}$ in hydroxylic solvents are higher than the previously reported quantum yields, where higher concentrations of 2 were used $\left.\left(\sim 10^{-3} \mathrm{M}\right)\right)^{3 b, 4 h}$ Probably excimer formation reduced the transformation quantum yields in the previous studies.
Table 1 Phototransformation quantum yields of chlorobenzene and 4-chloroanisole. The absorption at the excitation wavelength $(254 \mathrm{~nm})$ was $<0.02\left(5 \times 10^{-5}-1 \times 10^{-4} \mathrm{M}\right)$

\begin{tabular}{|c|c|c|c|c|}
\hline \multirow[b]{2}{*}{ Solvent } & \multicolumn{2}{|c|}{ Chlorobenzene (1) } & \multicolumn{2}{|c|}{ 4-Chloroanisole (2) } \\
\hline & Nitrogen & Air & Nitrogen & Air \\
\hline Cyclohexane & 0.53 & 0.068 & 0.72 & 0.051 \\
\hline Ethanol & 0.47 & 0.14 & 0.081 & 0.056 \\
\hline Methanol & 0.25 & 0.16 & 0.27 & 0.21 \\
\hline Glycerol/methanol $^{a}$ & - & 0.31 & - & 0.19 \\
\hline Acetonitrile & 0.16 & 0.13 & 0.015 & 0.014 \\
\hline
\end{tabular}

${ }^{a}$ Mixture 4:1. Standard errors are $\sim 15 \%$ for cyclohexane and $\sim 5 \%$ for the other solvents.

The degradation quantum yields of $\mathbf{1}$ and $\mathbf{2}$ depend on the hydrogen donor ability and polarity of the solvent in addition to the concentration of molecular oxygen (Table 1).

\section{Phototransformation products}

The photoproduct distributions (Scheme 2) depend on the solvent and on the presence of molecular oxygen. The dependence on oxygen indicates that, besides expected physical quenching (see Table 1), oxygen also interacts/reacts with the formed intermediates.

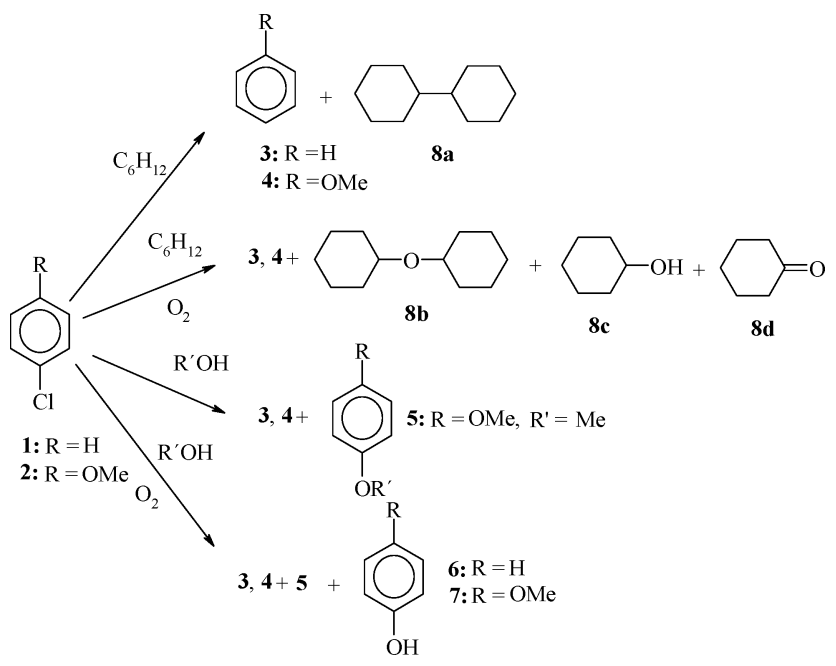

Scheme 2 Main phototransformation products.

Reduction is the main transformation process in all studied conditions which are in agreement with previous reports. ${ }^{3,4}$ In cyclohexane the phototransformation of $\mathbf{1}$ in air saturated solutions leads to the incorporation of oxygen into the solvent molecule, resulting in the formation of products $\mathbf{8 b}, \mathbf{8 c}$ and 8d. These products were also detected after photolysis of $\mathbf{2}$ in cyclohexane in the presence of oxygen. ${ }^{3 c}$ In hydroxylic solvents $\left(\mathrm{R}^{\prime} \mathrm{OH}\right)$ substitution also occurs to form products such as $\mathbf{4}$ and $\mathbf{6}$, for chlorobenzene (1) and 4-chloroanisole (2), respectively. Fig. 1 shows the effect of oxygen on the phototransformation products of $\mathbf{1}$ in methanol.

Under oxygen, besides reduction and substitution, other compounds with low retention times in HPLC, such as phenol (6) in the case of $\mathbf{1}$ and 4-hydroxyanisole (7) in the case of $\mathbf{2}$, are formed (Fig. 1 and 2). These polar products are not observed if 


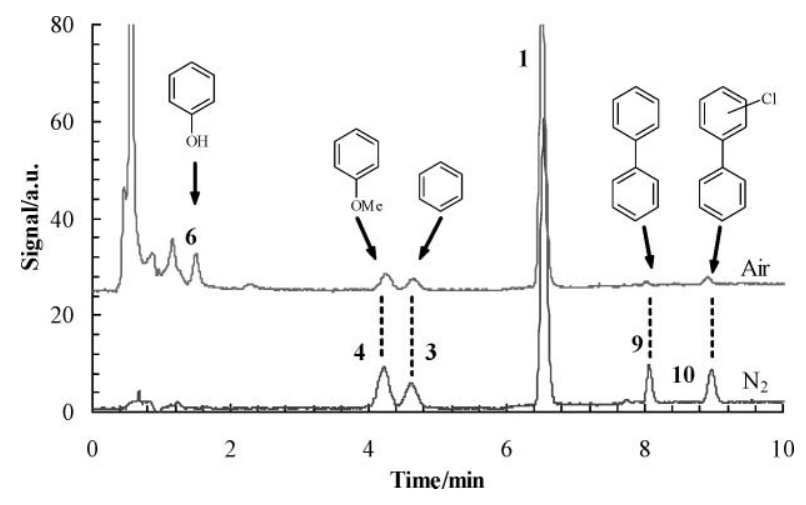

Fig. 1 HPLC traces of irradiated chlorobenzene (1) in methanol in the presence (top) and absence (bottom) of oxygen, detected at $254 \mathrm{~nm}$. Products 3, 4, 6, 9 and 10 are benzene, anisole, phenol, biphenyl and chlorobiphenyls, respectively. Initial concentration of $1: 2 \times 10^{-3} \mathrm{M}$.

the irradiation is performed under nitrogen atmosphere. In the case of $\mathbf{2}$ oxygen increases the ratio between $\mathbf{4}$ and $\mathbf{5}$, indicating that the substitution pathway in the case of $\mathbf{2}$ is more sensitive to the presence of oxygen than the reduction pathway. The effect of oxygen on the formation of new polar products (such as 7) and on the dimerization process (products $\mathbf{1 1}$ and $\mathbf{1 2}$ ) is, however, lower for 2 .

In acetonitrile solutions, the phototransformation is much slower, especially for compound 2 (see Table 1). However, the main reaction process still is the reductive dechlorination.

The photoproduct distributions also change with the concentration. For concentrations of $\mathbf{1}$ and $\mathbf{2}$ higher than $2 \times 10^{-3} \mathrm{M}$, dimerization becomes an important degradation path (Fig. 1 and 2 , products $9, \mathbf{1 0}, \mathbf{1 1}$, and $\mathbf{1 2}$ ).

The dimerization products of $\mathbf{2}$ were studied by LC-MS and show molecular ions with masses $\mathrm{m} / z=215$ for compounds $\mathbf{1 1}$ and 259 for compounds $\mathbf{1 2}\left(\mathrm{M}+\mathrm{H}^{+}\right.$, electro spray ionization) (Fig. 2, chromatograms $\mathrm{C}$ and D). These compounds, assigned to dimethoxybiphenyls and to chlorodimethoxybiphenyls, respectively, were also detected by GC-MS.

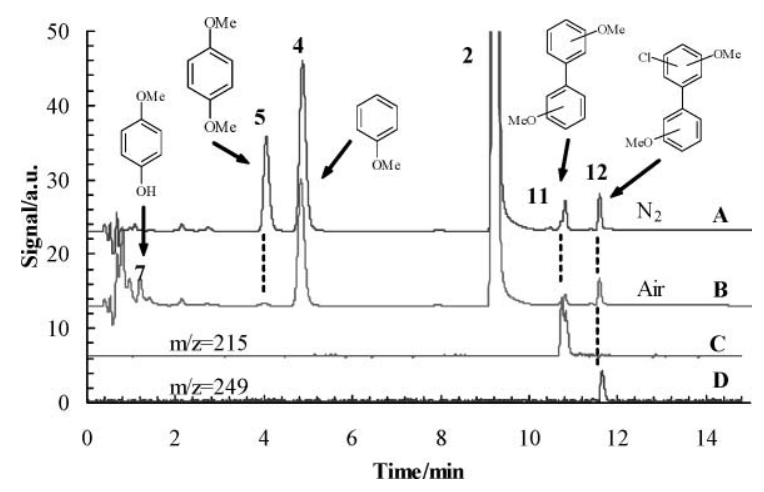

Fig. 2 HPLC traces of irradiated 4-chloranisole (2) in methanol with and without oxygen, detected at $275 \mathrm{~nm}$ (initial concentration of 1: $5 \times 10^{-3} \mathrm{M}$ ). Chromatograms $\mathrm{A}$ and $\mathrm{B}$ were obtained with the diode array detector of the LC; $\mathrm{C}$ and $\mathrm{D}$ are the chromatograms of single ion traces obtained for masses 215 and 259, respectively, for the sample irradiated under nitrogen (MS detector). Products 4, 5, 7, 11 and 12 are anisole, dimethoxyanisole, 4-hydroxyanisole, dimethoxybiphenyls and chlorodimethoxybiphenyls, respectively.
In the absence of oxygen (nitrogen atmosphere) an increase in the formation of biphenyl and chlorobiphenyls in comparison to air saturated conditions was observed. Oxygen should therefore interact with transients produced by primary photochemical processes, namely carbon centered radicals, forming new oxygenated intermediates which decrease the dimerization pathway. This effect is less pronounced for $\mathbf{2}$.

To better understand the genesis of the dimerization products, 1 and $\mathbf{2}$ were photolyzed in glycerol/methanol mixtures. Fig. 3 shows the photoproduct distribution obtained after irradiation of $\mathbf{2}$ in these conditions.

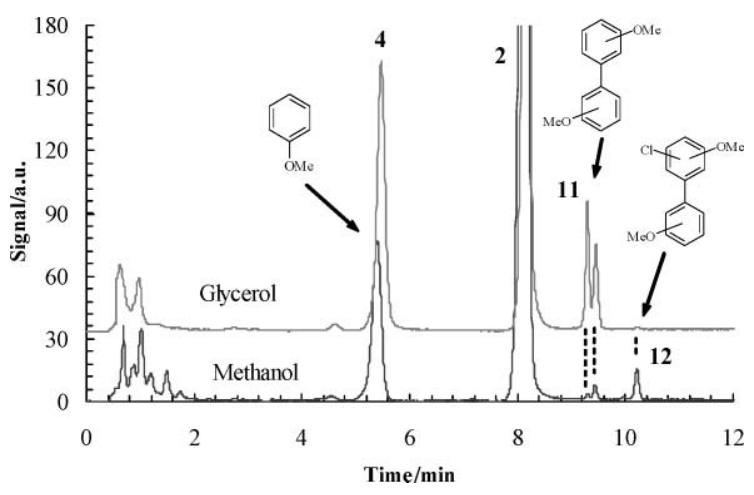

Fig. 3 HPLC traces of irradiated 4-chloranisole (2) in methanol (bottom) and in a glycerol/methanol (4:1) mixture (top), under air, detected at $275 \mathrm{~nm}$ (initial concentration of 2: $1 \times 10^{-2} \mathrm{M}$ ). Products 4, 11 and $\mathbf{1 2}$ are anisole, dimethoxybiphenyls and chlorodimethoxybiphenyls, respectively.

For comparison purposes, the product distribution in methanol obtained under air and with the same initial concentration is also shown. Glycerol quenches the formation of products $\mathbf{1 2}$ but, surprisingly promotes the formation of dimethoxybiphenyls (11). Glycerol reduces the diffusion and therefore is expected to quench the dimerization products formed by coupling of primary intermediates with ground state molecules. That is the case for chlorodimethoxybiphenyls (12). The reaction process that leads to products $\mathbf{1 1}$ is however different from that for 12. In these high viscosity systems the formation of dimethoxybiphenyls can be explained if some pre-association of two molecules of $\mathbf{2}$ in the ground state is assumed.

Irradiation of $\mathbf{1}$ in glycerol/methanol mixtures also promotes the formation of biphenyl (9) relatively to chlorobiphenyls (10), but the effect is less pronounced.

Most product assignments are based on comparison with authentic samples. Some products labeled $\mathbf{1 1}$ and $\mathbf{1 2}$ were assigned after examination of their mass spectra (GC-MS and LC-MS).

\section{Molar balances}

The molar balances (Table 2) confirm that reduction is the major degradation pathway in hydroxylic solvents. Oxygen influences both the reduction and the substitution pathways. In the case of $\mathbf{1}$ in methanol both reduction and substitution decrease about $50 \%$ when air is present. Substitution in $\mathbf{2}$ is, however, more sensitive to oxygen than reduction. In fact, as reported in previous studies, ${ }^{3 b}$ the substitution pathway almost vanishes in oxygen saturated conditions. 
Table 2 Photochemical reduction and substitution in hydroxylic solvents

\begin{tabular}{|c|c|c|c|c|}
\hline \multirow[b]{2}{*}{ Solvent } & \multicolumn{2}{|c|}{ Chlorobenzene $(\mathbf{1})^{b}$} & \multicolumn{2}{|c|}{ 4-Chloroanisole (2) ${ }^{c}$} \\
\hline & $\mathrm{ArH}(\%)$ & $\operatorname{ArOR}(\%)$ & $\mathrm{ArH}(\%)$ & $\operatorname{ArOR}(\%)$ \\
\hline Methanol $\left(\mathrm{N}_{2}\right)$ & 62 & 18 & 71 & 30 \\
\hline Methanol (Air) & 31 & 8 & 64 & 10 \\
\hline Ethanol $\left(\mathrm{N}_{2}\right)$ & 75 & 7 & 92 & ${ }^{d}$ \\
\hline Ethanol (Air) & 60 & 6 & 66 & $\ldots d$ \\
\hline Glycerol/methanol $^{a}$ (Air) & 38 & ${ }^{d}$ & 67 & $\complement^{d}$ \\
\hline
\end{tabular}

${ }^{a}$ Mixture 4:1. ${ }^{b}$ Concentrations $\sim 10^{-3}$ M. ${ }^{c}$ Concentrations $\sim 10^{-4}$ M. ${ }^{d}$ Not determined. Standard errors are $\sim 5 \%$, conversions $20-40 \%$.

\section{EPR}

The aryl chlorides $\mathbf{1}$ and $\mathbf{2}$ dissolved in several solvents (cyclohexane, acetonitrile, and methanol) were photolyzed inside the cavity of the EPR spectrometer. In the case of $\mathbf{1}$ and $\mathbf{2}$ in cyclohexane, a spectrum with six lines with a $\sim 22 \mathrm{G}$ distance was observed in the absence of oxygen (Fig. 4A). This spectrum was assigned to the solvent radical (cyclohexyl radical) based on the agreement with a literature spectrum, which was generated by electron beam irradiation of cyclohexane. ${ }^{10 a}$ Photolysis of the aryl chlorides 1 and 2 generates phenyl and methoxyphenyl radicals, respectively. Due to their high reactivity, these aryl radicals are not observable by EPR under our experimental conditions. $\mathrm{H}$-abstraction from the solvent dominates. When oxygen is present during the photolysis a broad signal is detected (Fig. 4B) for both substrates (1 and $\mathbf{2}$ ). This broad signal was assigned to peroxyl radicals, which were generated by scavenging of radicals (cyclohexyl radicals and aryl radicals) by oxygen. The presence of cyclohexylperoxy radicals is in agreement with the observation of the photoproducts $8 \mathbf{8 b}, \mathbf{8 c}$ and $\mathbf{8 d}$ (Scheme 2), in the presence of oxygen.

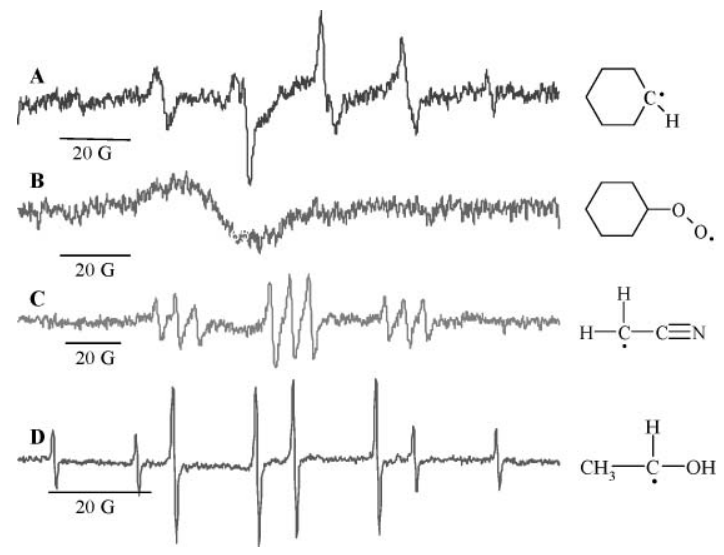

Fig. 4 EPR Spectra of irradiated chlorobenzene (1) in cyclohexane without (A) and with oxygen (B), of irradiated chlorobenzene (1) in acetonitrile without oxygen (C) and of irradiated 4-chloroanisole (2) in methanol also without oxygen (D).

If acetonitrile is used as solvent, photolysis of $\mathbf{1}$ under argon atmosphere generated an EPR spectrum consistent with the solvent radical (Fig. 4C) ${ }^{10 b}$ However, photolysis of 2 in acetonitrile did not yield a detectable EPR signal.

Photolysis of $\mathbf{1}$ and $\mathbf{2}$ in ethanol generated the solvent radical (methylketyl radical; Fig. 4D). ${ }^{10 c}$ The generation of the solvent radical is in agreement with the photoproduct distributions and mass balance data and strongly support the reduction pathway in these systems. The presence of oxygen quenches both the acetonitrile and ethanol radicals. However, the EPR signals of the corresponding peroxyl radicals were not clearly observable as in the case of cyclohexane as solvent.

\section{LFP}

Laser flash photolysis studies with excitation at $266 \mathrm{~nm}$ of argon, oxygen and air saturated solutions of $\mathbf{1}$ and $\mathbf{2}$ were performed in several solvents. In cyclohexane, intense transient absorption bands centered at $300 \mathrm{~nm}$ (Fig. 5 inset) and $330 \mathrm{~nm}$ were obtained for $\mathbf{1}$ and $\mathbf{2}$, respectively, in argon saturated solutions. These transients are quenched by oxygen with rate constants of $k_{\mathrm{o} 2} \approx 4 \times 10^{9} \mathrm{M}^{-1} \mathrm{~s}^{-1}$ and were assigned to the corresponding triplet states of $\mathbf{1}$ and 2. $^{3 c, 11}$

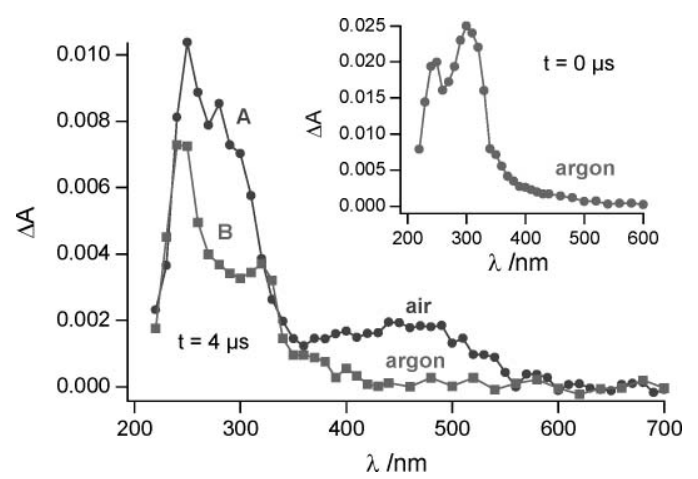

Fig. 5 Transient absorption spectra of chlorobenzene (1) in cyclohexane, with (A) and without (B) oxygen, observed $4 \mu$ s after the laser pulse $(266 \mathrm{~nm})$. The inset shows the transient absorption spectrum in the absence of oxygen at the end of the laser pulse.

In the presence of oxygen a transient absorption band centered at $460 \mathrm{~nm}$ that increases linearly with the laser power, is observed for $\mathbf{1}$ in cyclohexane (Fig. 5). A similar transient absorption (centered at $550 \mathrm{~nm}$ ) was observed for $\mathbf{2}$ in cyclohexane, which was previously studied by us in the presence of oxygen, ${ }^{3 c}$ and the absorption was assigned to 4-methoxyphenylperoxyl radicals.

Phenylperoxyl radicals have been produced by pulse radiolysis in the presence of molecular oxygen..$^{12}$ The mechanism of phenylperoxy radical formation involves a reductive dehalogenation of the respective halobenzene, followed by the rapid reaction of the phenyl radical with oxygen. These radicals show broad absorption bands in the visible region of the spectrum. The observed transient absorption centered at $460 \mathrm{~nm}$ (Fig. 5A) was therefore assigned to the phenylperoxyl radical. The phenylperoxyl radicals were also observed for $\mathbf{1}$ in acetonitrile and ethanol (Figure S-1, ethanol, ESI $\dagger$ ). For 2 the transient absorption spectra in polar solvents (acetonitrile and hydroxylic solvents) differ significantly from the spectra in non polar solvents (cyclohexane). In acetonitrile, methanol and ethanol transient absorption between 400 and $500 \mathrm{~nm}$ were observed (Figure S-2 left, ethanol, ESI $\dagger$ ), which were assigned to ionic intermediates. ${ }^{4 b, h}$ The intensity $(\Delta \mathrm{A})$, decay kinetics and spectral distributions of the detected transient absorption bands are independent on the oxygen concentration (Figure S-2 right, ethanol, ESI $\dagger$ ). The low reactivity of the transients with oxygen confirms the ionic character of the detected species. ${ }^{13}$ Because the absorption of 4-methoxyphenylperoxyl 
radicals extends above $500 \mathrm{~nm}$, their detection in a spectral region that does not overlap with that of the ionic transients is possible. In the presence of oxygen a weak transient absorption signal was indeed observed at $550 \mathrm{~nm}$. This result indicates that the methoxyphenylperoxyl radical formation is less efficient in hydroxylic solvents, such as ethanol.

\section{Mechanism}

The internal heavy atom effect in aryl chlorides makes the $\mathrm{S}_{1} \rightarrow \mathrm{T}_{1}$ intersystem crossing very efficient and therefore, triplet states are expected to have a dominant role in the phototransformations. Since homolytic cleavage is a likely process from triplet states, the primary photochemical process (PPP) step should be the homolytic cleavage of the $\mathrm{C}-\mathrm{Cl}$ bond and formation of the triplet radical pair in the solvent cage (Scheme 3). Therefore, diffusion from the solvent cage (Scheme 3, path A) followed by hydrogen abstraction by phenyl radicals should be the main reaction pathway (Scheme 3, path B). The involvement of phenyl radicals is in fact reported to be the only reduction process in non-polar solvents. ${ }^{3}$ The homolytic cleavage of the $\mathrm{C}-\mathrm{Cl}$ bond is however difficult to follow by flash photolysis, because phenyl radicals absorb in the same spectral region of the parent compound. ${ }^{6}$ However, the phototransformation in the presence of oxygen leads to the reaction of phenyl radicals with oxygen (Scheme 3, reaction $\mathbf{C}$ ). The generated phenylperoxy radicals absorb at longer wavelengths $(>400 \mathrm{~nm})$, which provides a means of following this reaction by LFP. Oxygen can also quench the excited states involved in the formation of phenyl radicals and therefore, only when the rate constant of homolytic fission is higher or at least similar to that of the oxygen quenching, the above procedure will allow for the detection of the homolytic cleavage.

The rate constants of hydrogen abstraction of the phenyl radical from hydrocarbons $\left(\sim 10^{6} \mathrm{M}^{-1} \mathrm{~s}^{-1}\right.$; Scheme 3 , reaction $\left.\mathbf{B}\right)$ are three orders of magnitude lower than the bimolecular rate constant for the reaction of phenyl radicals with oxygen $\left(\sim 10^{9} \mathrm{M}^{-1} \mathrm{~s}^{-1}\right){ }^{12}$ Since concentration of dissolved oxygen in organic solvents is $\sim 10^{-3} \mathrm{M}$ under air saturated condition, ${ }^{14}$ the rates of reactions $\mathbf{B}$ and $\mathbf{C}$ are expected to compete under air saturated conditions. An increase in the concentration of oxygen should favor process $\mathbf{C}$ (under oxygen saturation the concentration is about five times higher than in air saturated conditions ${ }^{14}$ ). Therefore, performing experiments under different concentrations of oxygen is helpful to assign reaction pathways and reaction products.

The main reaction of peroxyl radicals is known ${ }^{15}$ and involves the coupling of two peroxyl radicals. Products 8b, 8c and 8d (Scheme 2), result from the coupling of cyclohexylperoxyl radicals followed by $\mathrm{O}_{2}$ elimination. Phenylperoxyl radicals are expected to undergo similar coupling and form phenoxyl radicals (Scheme 3, reaction D), ${ }^{12}$ which lead to 6 and 7 (see Scheme 2) after $\mathrm{O}_{2}$ elimination and hydrogen abstraction. For low concentrations of $\mathbf{1}$ and $\mathbf{2}$ the weight of this bimolecular process decreases and the back reaction to the phenyl radical (Scheme 3, reaction $\mathbf{E}$ ) should gain importance.

In hydroxylic solvents (e.g., ethanol) both compounds undergo substitution and reduction. In the case of 1 no absorption bands assignable to ionic transients were detected. For $\mathbf{2}$ the detected ionic intermediates are insensitive to oxygen, but the substitution pathway is quenched by oxygen. Reduction is the main reaction process for both substrates, but the detected absorption of the 4-methoxyperoxyl radicals is weak. How can we rationalize mechanistically these findings?

Probing the reaction with oxygen allowed us to find a simple answer. First, the detected ionic transients must show little contribution to the final products, which are quenched by oxygen; the precursor of substitution should therefore be phenyl cations absorbing below $300 \mathrm{~nm} \cdot{ }^{13}$ Second, the precursor of reduction must be the phenyl radical; the formation of anisole through phenyl cations in the case of $\mathbf{2}$ was not observed ${ }^{13}$ and the possible formation of anisole after reaction of the 4-methoxyphenyl cation with ground state of $\mathbf{2}^{4 h}$ is excluded at low concentrations $\left(<10^{-4} \mathrm{M}\right)$. Third, both reaction pathways can be rationalized if we consider that homolytic cleavage and in cage radical pair formation is the general first transformation step. Furthermore, in polar solvents electron transfer within the caged radical pair leads to the formation of the ion pair (Scheme 3, reaction $\mathbf{F}$ ). This latter key reaction step was first proposed for alkyl halides ${ }^{16}$ and then extended to chlorobenzene ${ }^{4 c}$ and 4-chlorobiphenyl ${ }^{4 d, e}$ in

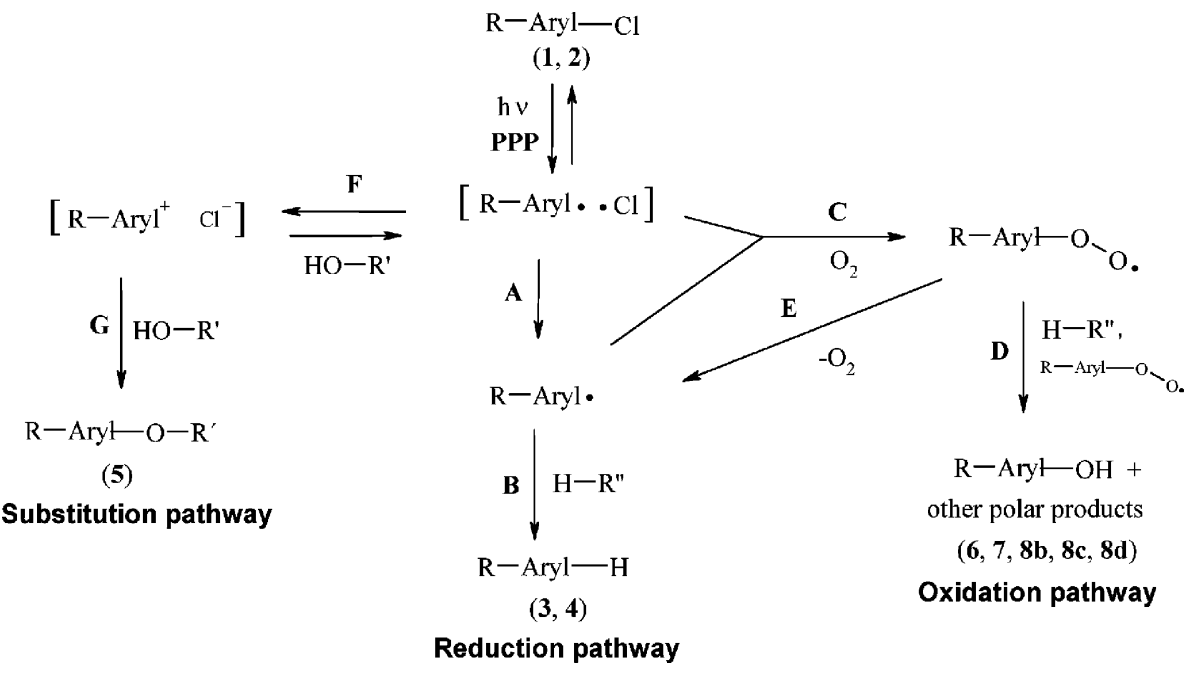

Scheme 3 Main phototransformation pathways of aryl chlorides. 
aqueous solutions. As will be shown below, the generalization of this transformation pathway provides a means to draw a unified picture of the photoreactivity of aryl chlorides and other aryl halides in a wide range of conditions.

For the compounds studied, the electron transfer and consequent formation of ionic species is expected to occur mainly in hydroxylic solvents. The so-formed phenyl cation can then lead to substitution (Scheme 3, reaction G). ${ }^{4 g, h}$ In the absence of oxygen the weight of substitution relative to reduction depends on the competition between electron transfer (reaction F) and cage escape/hydrogen abstraction (reaction $\mathbf{A} / \mathbf{B}$ ). When oxygen is present a third reaction channel is opened for the radical pair/phenyl radicals: phenylperoxyl radical formation (reaction C). This way, the increase in the concentration of dissolved oxygen also increases the formation of the peroxyl radical and consequently lowers the weight of the substitution process. The phenylperoxyl radicals can also undergo back reaction and reform the phenyl radical. ${ }^{3 \mathrm{c}}$ However, after reaction $\mathbf{E}$, the cation formation according to reaction $\mathbf{F}$ is no longer possible (the phenyl-chlorine radical pair is lost) and only reactions $\mathbf{B}$ from the phenyl radical and $\mathbf{C}$ occur. This way, oxygen quenches the substitution pathway $(\mathbf{F})$ and leaves the reduction pathway $(\mathbf{B})$.

For higher concentrations of $\mathbf{1}$ and $\mathbf{2}$, the phenyl cation and the phenyl radical can couple to ground state molecules and form dimerization products. ${ }^{4 g, h, 17}$ The formation of dimers/excimers can also lead to these products. Triplet complexes (excimers) of $\mathbf{2}$ have already been detected by flash photolysis and reported to form radical cations. ${ }^{4 b}$ Radical cations of $\mathbf{2}$ can also dimerize or react with ground state molecules and form dimethoxybiphenyls. ${ }^{18}$ The involvement of dimers/excimers in the formation of the carboncarbon bond deserves further studies.

\section{Conclusions}

The effect of molecular oxygen on the photochemical transformations of aryl chlorides can be used as a powerful tool to probe their elementary reactions. The effect of oxygen on the photoreactivity of aryl chlorides allows for a unified picture of the reactivity of these compounds in a wide range of conditions (Scheme 3). The main primary reaction process is the homolytic cleavage of the $\mathrm{C}-\mathrm{Cl}$ bond and consequent formation of the triplet radical pair in the solvent cage. Hydrogen abstraction leads to reduction, and electron transfer followed by reaction of the phenyl cation with a nucleophile leads to substitution. The reaction of the radical pair with oxygen forms phenylperoxyl radicals and extends the transient absorption to the visible region of the spectrum, allowing the detection of this primary reaction step by LFP. Phenylperoxyl radicals undergo dimerization by coupling to each other and form polar products or undergo back reaction to the phenyl radicals.

For concentrations higher than $10^{-3} \mathrm{M}$ of the aryl chlorides, dimerization becomes an important transformation process. The dimerization products are formed by reaction of the primary intermediates with ground state aryl chlorides but might also occur through excimer formation. The importance of the dimerization reactions in synthesis, especially the possible involvement of dimers/excimers in the formation of the carbon-carbon bond, the photochemical transformation and role of oxygen in these conditions should be further explored.

\section{Acknowledgements}

J.P.S. thanks the Fundação Calouste Gulbenkian for a travel grant and the Luso-American Foundation for financial support (project FLAD/NSF ref. 600-13/2006). The authors at Columbia thank the National Science Foundation for support through grants NSFCHE 04-15516 and NSF-CHE 07-17518.

\section{Notes and references}

1 (a) R. S. Davidson, J. W. Goodin and G. Kemp, The photochemistry of aryl halides and related-compounds, Adv. Phys. Org. Chem., 1984, 20, 191-233; (b) J. Cornelisse and E. Havinga, Photosubstitution reactions of aromatic-compounds, Chem. Rev., 1975, 75, 353-388.

2 (a) K.-L. Han and G.-Z. He, Photochemistry of aryl halides: photodissociation dynamics, J. Photochem. Photobiol. C: Photochem. Rev., 2007, 8, 55-66; (b) M. Fagnoni and A. Albini, Arylation reactions: the photo-SN(1) path via phenyl cation as an alternative to metal catalysis, Acc. Chem. Res., 2005, 38, 713-721.

3 (a) N. J. Bunce, J. P. Bergsma, M. D. Bersma, W. De Graff, Y. Kumar and L. Ravanal, Structure and mechanism in the photoreduction of aryl chlorides in alkane solvents, J. Org. Chem., 1980, 45, 3708-3713; (b) J. R. Siegman and J. J. Houser, Photodehalogenation of the monochloroand monofluoroanisoles, J. Org. Chem., 1982, 47, 2773-2779; (c) J. P. Da Silva, L. F. Vieira Ferreira, I. F. Machado and A. M. Da Silva, Photolysis of 4-chloroanisole in the presence of oxygen. Formation of the 4-methoxyphenylperoxyl radical, J. Photochem. Photobiol. A: Chem., 2006, 182, 88-92.

4 (a) J. Den Heijer, O. B. Shadid, J. Cornelisse and E. Havinga, Photoreactions of aromatic compounds-XXXV. Nucleophilic, photosubstitution of methoxy substituted aromatic compounds. Monophotonic ionization of the triplet state, Tetrahedron, 1977, 33, 779-786; (b) H. Lemmetyinen, J. Konijnenberg, J. Cornelisse and C. A. Varma, Laser spectroscopic study of the nucleophilic photosubstitution of 4chloroanisole and 4-fluoroanisole in aqueous solutions, J. Photochem., 1985, 30, 315-338; (c) D. Dulin, H. Drossman and T. Mill, Products and quantum yields for photolysis of chloroaromatics in water, Environ. Sci. Technol., 1986, 20, 72-77; (d) T. Moore and R. M. Pagni, Unusual photochemistry of 4-chlorobiphenyl in water, J. Org. Chem., 1987, 52, 770-773; (e) J. Orvis, J. Weiss and R. M. Pagni, Further studies on the photoisomerization and hydrolysis of chlorobiphenyls in water. Common ion effect in the photohydrolysis of 4-chlorobiphenyl, $J$. Org. Chem., 1991, 56, 1851-1857; ( $f$ ) G. Grabner, C. Richard and G. Köhler, Formation and reactivity of 4-oxocyclohexa-2,5-dienyliden in the photolysis of 4-chlorophenol in aqueous solution at room temperature, J. Am. Chem. Soc., 1994, 116, 11470-11480; $(g)$ S. Protti, M. Fagnoni, M. Mella and A. Albini, Aryl cations from aromatic halides. Photogeneration and reactivity of 4-hydroxy(methoxy)phenyl cation, J. Org. Chem., 2004, 69, 3465-3473; (h) I. Manet, S. Monti, G. Grabner, S. Protti, D. Dondi, V. Dichiarante, M. Fagnoni and A. Albini, Revealing phenylium, phenonium, vinylenephenonium, and benzenium ions in solution, Chem. Eur. J., 2008, 14, 1029-1039.

5 (a) M. Rasmusson, R. Lindh, N. Lascoux, A. N. Tarnovsky, M. Kadi, O. Kühn, V. Sundström and E. Akesson, Photodissociation of bromobenzene in solution, Chem. Phys. Lett., 2003, 367, 759-766; (b) Y.-J. Liu, P. Persson and S. Lunell, Multiference calculations of the phosphorescence and photodissociation of chlorobenzene, J. Chem. Phys., 2004, 121, 11000-11006.

6 (a) B. Cercek and M. Kongshaug, Phenyl and hydroxyphenyl radicals. Pulse radiolysis study of their spectra and reactivity, J. Phys. Chem., 1970, 74, 4319-4322; (b) N. Ikeda, N. Nakashima and K. Yoshihara, Observation of the ultraviolet-absorption spectrum of phenyl radical in the gas phase, J. Am. Chem. Soc., 1985, 107, 3381-3382; (c) J. G. Radziszewski, Electron absorption spectrum of phenyl radical, Chem. Phys. Lett., 1999, 301, 565-570.

7 A. Leifer, The Kinetics of Environmental Aquatic Photochemistry. Theory and Practice; ACS Professional Reference Book, Maple Press Company, York, PA, 1988.

8 J. P. Da Silva, L. F. Vieira, Ferreira and A. M. Da Silva, Aqueous photochemistry of pesticides triadimefon and triadimenol, J. Photochem. Photobiol. A: Chem., 2003, 154, 293-298.

9 Y. Yagci, S. Jockusch and N. J. Turro, Mechanism of photoinduced step polymerization of thiophene by onium salts: Reaction of 
phenyliodonium and diphenylsulfinium radical cations with thiophene, Macromolecules, 2007, 40, 4481-4485.

10 (a) R. W. Fessenden and R. H. Schuler, Electron spin resonance studies of transient alkyl radicals, J. Chem. Phys., 1963, 39, 2147-2195; (b) P. Smith, R. A. Kaba, T. C. Smith, J. T. Pearson and P. B. Wood, EPR study of radicals formed from aliphatic nitriles, J. Magn. Res., 1975, 18, 254-264; (c) W. T. Dixon and R. O. C. Norman, Electron spin resonance of oxidation. Alcohols, J. Chem. Soc., 1963, 31193124.

11 C. M. Previtali and T. W. Ebbesen, Photophysics of chlorobenzene in cyclohexane, J. Photochem., 1984, 27, 9-15.

12 (a) G. I. Khaikin, Z. B. Alfassi and P. Neta, Arylperoxyl radicals formation, absorption spectra, and reactivity in aqueous-alcohol solutions, J. Phys. Chem., 1995, 99, 265-268; (b) G. I. Khaikin, Z. B. Alfassi and P. Neta, Inter- and intramolecular redox reactions of substituted phenylperoxyl raicals in aqueous solutions, J. Phys. Chem., 1995, 99, 16722-16726; (c) X. W. Fang, R. Mertens and C. J. von Sonntag, Pulse radiolysis of aryl bromides in aqueous solutions. Some properties of aryl and arylperoxyl radicals, J. Chem. Soc. Perkin Trans., 1995, 2, 1033-1036.
13 S. Steenken, M. Ashokkumar, P. Maruthamuthu and R. A. McClelland, Making photochemically generated phenyl cations visible by addition to aromatics: production of phenylcyclohexadienyl cations and their reactions with bases/nucleophiles, J. Am. Chem. Soc., 1998, 120, 11925-11931.

14 S. L. Murov, Handbook of Photochemistry, M. Dekker, Inc., New York, 1973.

15 (a) G. A. Russell, Deuterium-isotope effects in the autoxidation of aralkyl hydrocarbons. Mechanism of the interaction of peroxy radicals, J. Am. Chem. Soc., 1957, 79, 3871-3877; (b) J. E. Bennett, Kinetic electron paramagnetic resonance study of the reactions of t-butylperoxyl radicals in aqueous solution, J. Chem. Soc., Faraday Trans., 1990, 86, 3247-3252.

16 P. J. Kropp, Photobehavior of alkyl halides in solution: radical, carbocation, and carbene intermediates, Acc. Chem. Res., 1984, 17, 131-137.

17 J. C. Scaiano and L. C. Stewart, Phenyl radical kinetics, J. Am. Chem. Soc., 1983, 105, 3609-3614.

18 N. Govindaraj, M. M. Mortland and S. A. Boyd, Single electron transfer mechanism of oxidative dechlorination of 4-chloroanisole on copper(II)-smectite, Environ. Sci. Technol., 1987, 21, 1119-1123. 